\title{
Magnetic resonance cholangiopancreatography (MRCP) using new negative per-oral contrast agent based on superparamagnetic iron oxide nanoparticles for extrahepatic biliary duct visualization in liver cirrhosis
}

\author{
Katerina Polakovaa, Ingrid Mocikova ${ }^{b, d}$, Dana Purovac, Pavel Tucekc, Pavel Novak ${ }^{b}$, Katerina Novotnad, Izak Nikod, \\ Radoslav Bielik ${ }^{\mathrm{d}}$, Radek Zborila ${ }^{\mathrm{a}}$, Miroslav Hermane
}

\begin{abstract}
Background and Aims. Magnetic resonance cholangiopancreatography (MRCP) is often used for imaging of the biliary tree and is required by surgeons before liver transplantation. Advanced liver cirrhosis and ascites in patients however present diagnostic problems for MRCP. The aim of this study was to find out if the use of our negative per-oral contrast agent containing superparamagnetic iron oxide nanoparticles (SPIO) in MRCP is helpful for imaging of hepatobiliary tree in patients with liver cirrhosis.

Methods. Forty patients with liver cirrhosis were examined on a 1.5 T MR unit using standard MRCP protocol. Twenty patients (group A) underwent MRCP after administration of per-oral SPIO contrast agent 30 min before examination. In group B, twenty patients were examined without per-oral bowel preparation. Ascites was present in eleven patients from group $A$ and in thirteen patients in group $B$. Four radiologists analyzed MR images for visibility and delineation of the biliary tree. $x^{2}$ tests were used for comparison of the visibility of intrahepatic and extrahepatic biliary ducts in patients with and without ascites.

Results. Better extrahepatic biliary duct visualization and visibility of extraluminal pathologies in patients with ascites was proved after administration of SPIO contrast agent. No statistically significant difference between group A and B was found for visualization of extrahepatic biliary ducts in patients without ascites. Delineation of intrahepatic biliary ducts was independent on bowel preparation.
\end{abstract}

Conclusions. Application of our negative per-oral SPIO contrast agent before MRCP improves the visualization of extrahepatic biliary ducts in patients with ascites which is helpful during the liver surgery, mainly in liver transplantation.

Key words: superparamagnetic iron oxide (SPIO) nanoparticles, per-oral MR contrast agent, magnetic resonance cholangiopancreatography (MRCP), liver transplantation, cirrhosis, ascites

Received: March 16, 2016; Accepted with revision: August 23, 2016; Available online: September 15, 2016 http://dx.doi.org/10.5507/bp.2016.046

${ }^{a}$ Regional Centre of Advanced Technologies and Materials, Departments of Physical Chemistry and Experimental Physics, Faculty of Science, Palacky University Olomouc, Czech Republic

'Medihope s.r.o., Mathonova 291/1, 79604 Prostejov, Czech Republic

'Department of Geoinformatics, Faculty of Science, Palacky University Olomouc, Czech Republic

${ }^{d}$ Department of Radiology, F.D.Roosevelt Faculty Hospital, Banska Bystrica, Slovak Republic

${ }^{e}$ Department of Radiology, Faculty of Medicine and Dentistry, Palacky University Olomouc, Czech Republic

Corresponding author: Katerina Polakova, e-mail:dr.kacka.polakova@gmail.com

\section{INTRODUCTION}

Cirrhosis is a chronic liver disease characterized by the deposition of fibrous tissue within the liver. From a clinical point of view, there is a progressive loss of liver function and increasing risk of liver cancer ${ }^{1}$. Liver transplantation is the only possible method of treatment in end-stage liver cirrhosis. Correct timing of liver transplantation can reduce mortality and also improve post-transplant survival. Various imaging modalities are available for imaging of the biliary tree and pancreatic duct such as ultrasound (US), endoscopic retrograde cholangiopancreatography (ERCP), multislice computed tomography (CT) and magnetic resonance cholangiopancreatography (MRCP). MRCP is a non-invasive alternative technique to ERCP for imaging the biliary anatomy and pathology of the intrahepatic and extrahepatic bile ducts in various biliary diseases as well as in liver cirrhosis ${ }^{2}$. Advanced stages of liver cirrhosis are often combined with the presence of ascites of various amounts as a result of advanced portal vein hypertension. In ascites, exact visualization of the biliary tree and bowel wall is generally more difficult. Advanced ascites combined with bowel wall edema (as a result of advanced portal vein hypertension, beside other pathologies such as varices, portal thrombosis, weakness, breathing problems, coagulation disorders, etc.) complicate imaging of the extrahepatic biliary ducts.

The detection of abdominal pathology and particularly biliary tree pathology is crucial not only for surgical but also for interventional planning ${ }^{2}$. MRCP is mandatory in the list of examinations before liver transplantation. MRCP is a totally noninvasive, safe and well tolerated examination and regularly used before liver transplantation for accurate biliary tree analysis. However some diagnos- 
tic problems exist with MRCP in cases of advanced liver cirrhosis and ascites (one of the patterns of the cirrhosis decompensation). Ascites obfuscate assessment of extrahepatic biliary ducts, detection of the bowel loops and pathologies of the bowel wall and extraluminal conditions. Generally, MRCP is performed without any special preparation, but the presence of ascites prompted radiologists to suppress the signal intensity of the bowel lumen. As recently described, one possibility is filling the bowel lumen with superparamagnetic iron oxide (SPIO) based negative per-oral contrast agent ${ }^{3-5}$, which allows better differentiation of the extrahepatic biliary tree. The need for bowel opacification with the use of oral contrast medium is mandatory to differentiate between collapsed or fluid-filled bowel loops, extrahepatic biliary ducts, intra-abdominal organs, ascites and pathological lesions.

In MR enterography and MR enteroclysis, three main groups of intraluminal contrast agents are generally used. These affect the signal intensity (hyper- or hypointense) of bowel lumen and/or bowel wall called positive, biphasic and negative per-oral contrast agents. SPIO containing maghemite $\left(\gamma-\mathrm{Fe}_{2} \mathrm{O}_{3}\right)$ and/or magnetite $\left(\mathrm{Fe}_{3} \mathrm{O}_{4}\right)$ iron oxide cores are strong proton enhancers of surrounding water containing tissue providing a negative signal in T2-weighted MR images. For per-oral application they are usually incorporated into a biocompatible and insoluble matrix or shell such as polystyrene (commercial Abdoscan), siloxan (commercial Lumirem) ( ref. $^{6}$ ) or bentonite ${ }^{5}$. These SPIO per-oral contrast agents enable decrease in noise and motion artifacts related to bowel peristalsis and help us to improve the detection of intramural edema and extramural complications (necrosis, fat edema, mesenterial edema, abscess formation or fistulas) $\left(\right.$ ref. $\left.^{7,8}\right)$. Ascites and bowel wall edema are displayed with high signal intensity in T2-weighted images and low in T1-weighted images, which is the same as for biphasic contrast agents. While signal intensity of positive contrast agent is high in T2- and T1-weighted images, on the other hand negative contrast agents are depicted as hypointense in T2- and T1-weighted images ${ }^{7}$. Therefore, application of negative contrast agents is the best choice for effective visualization of the above-mentioned problem parts of the abdominal cavity.

As there are no published data so far, we decided to evaluate our per-oral negative SPIO based contrast agent in patients with liver cirrhosis (with/ without ascites) by MRCP modality. In this study, we evaluated the results of MRCP investigations in patients with advanced liver cirrhosis, after administration of experimentally prepared SPIO per-oral contrast agent containing maghemite nanoparticles in bentonite matrix.

\section{METHODS}

\section{Patients}

This study was approved by the institutional Ethics Committee. A total of 40 patients ( 15 women and 25 men), aged 20-88 years, were included. The inclusion criteria were established as follows: the presence and confirmation of liver cirrhosis and indication for MRCP and signed informed consent before examination. MRCP was indicated as standard examination in patients with liver cirrhosis of any degree or before planned liver transplantation. Liver cirrhosis in patients with planned liver transplantation was scored with model for end-stage liver disease (MELD) score by clinicians ${ }^{9}$ and this fact was known to the radiologists before MRCP. Patients not planned for liver transplantation had no MELD score. All patients underwent ultrasound examination and the presence/absence of ascites was known prior to MRCP. Patients were divided into two groups according to bowel preparation before MRCP.

Group A consisted of 20 consecutive patients (10 women and 10 men). They were examined by MRCP after administration of per-oral negative SPIO contrast agent. Twelve of them were tested immediately prior to liver transplantation and eleven patients suffered from ascites. Group B consisted of the next 20 consecutive patients ( 5 women and 15 men). They were examined by MRCP without bowel preparation. Fourteen patients were before liver transplantation and thirteen had ascites.

\section{Imaging protocol}

All examinations were performed on a $1.5 \mathrm{~T}$ MRI unit (Signa Horizon Lx, General Electric, Milwaukee, Wisconsin, USA), with body phased array coil, in prone position. Protocol of MRCP: localizer; axial and coronal planes 2D T2 SSFSE TR $6000 \mathrm{~ms}$, TE $500 \mathrm{~ms}$, bandwidth 31.25 , matrix $384 \times 320$, FOV $36 \mathrm{~cm}$; coronal plane $3 \mathrm{D}$ FIESTA; 2D MRCP - slice thickness $20 \mathrm{~mm}$, spacing 0, number of slices 14 and more, without intravenous contrast agent or spasmolytic.

All image analyses were analyzed on the Advantage Windows workstation, software version 4.5 (General Electric, Milwaukee, Wisconsin, USA).

Patients in group A were examined after administration of $500 \mathrm{~mL}$ of per-oral negative SPIO contrast suspension 30 minutes before MRCP investigation. Patients in group B were examined without any per-oral preparation.

\section{Negative SPIO per-oral contrast agent}

The experimental per-oral suspension (final volume of $500 \mathrm{~mL}$ ) consisted of maghemite SPIO nanoparticles ( $400 \mathrm{mg}$ of gamma- $\mathrm{Fe}_{2} \mathrm{O}_{3}$ ) incorporated in bentonite matrix ( $2 \mathrm{~g}$ ) and 1 packet of polyethylene glycol 4000 (Macrogol) dissolved in $250 \mathrm{ml}$ of fruit apple/carrot juice (HAMI, Nutricia, CZ) mixed with water $(250 \mathrm{~mL})$. The application of our contrast agent has been succesfully tested previously in abdominal MRs (ref. ${ }^{5}$ ).

\section{Analysis}

Filled questionnaires from all four radiologists ( $1, \mathrm{p} 2$, p3, p4) were transformed into the dataset and statistically evaluated.

Four radiologists $(\mathrm{p} 1=\mathrm{RB}, \mathrm{p} 2=\mathrm{PN}, \mathrm{p} 3=\mathrm{NI}, \mathrm{p} 4=\mathrm{KN})$ performed a blind review of all MRCP investigations in both groups of patients. Of these, p1, p3, p4 were trained in gastrointestinal MRI. p2 was experienced in MR gen- 
erally, without special workout in gastrointestinal MR imaging.

After MRCP examination of each patient in both groups, all four radiologists completed a questionnaire on the quality of the diagnosed abdominal part evaluating:

(i) visualization of intrahepatic biliary tree: 1) excellent (80-100\%), 2) sufficient $(50-80 \%), 3)$ insufficient (less than 50\%), 4) impossible to evaluate;

(ii) visualization of extrahepatic biliary ducts: 1) excellent $(80-100 \%), 2)$ sufficient $(50-80 \%), 3)$ insufficient (less than 50\%), 4) impossible to evaluate;

(iii) absence/presence of bowel wall edema: 1) yes, 2) no;

(iv) presence of extraluminal bowel pathology: 1) ascites, 2) varices, 3) other pathologies;

(v) presence and amount of ascites: 1) absent, 2) mild amount, 3) advanced ascites; Advanced ascites was defined as a large amount of free intra-abdominal fluid causing a pressure and rotation of intra-abdominal organs (difficult breath intake within breathold, great enlargement of the peritoneal cavity). Mild ascites was defined as the presence of free abdominal fluid without the above described symptoms.

(vi) in group A distribution of bowel luminal filling of negative SPIO contrast solution.

\section{Statistics}

The $\chi^{2}$ test was used for patients with and without ascites, separately for comparison of intrahepatic and extrahepatic bile ducts.

The same test was used for evaluating categorical variables by all 4 radiologists: $P_{1}, P_{2}, P_{3}, P_{4}$. The level of significance was $P \leq 0.05$. Analysis was performed with the program STATISTICA, 10 (Statsoft Inc., Tulsa, OK, USA). The graphical visualization of categorical dates was performed by Mosaic plots.

\section{RESULTS}

It was established that visualization of extrahepatic biliary ducts in patients with ascites prepared with negative per-oral SPIO contrast agent was found to be significantly better according to all four radiologists $\left(P_{1}=\right.$ $0.000039, P_{2}=0.00039, P_{3}=0.00279, P_{4}=0.00002$ ) (see Fig. 1. and images of patient investigated without and with negative contrast agent, Fig. 2 and 3, respectively). In patients without ascites, visualization of extrahepatic biliary ducts was independent of bowel preparation according to all four radiologists $\left(P_{1}=0.102, P_{2}=0.116, P_{3}\right.$ $=0.976, P_{4}=0.413$ ).

Visualization of intrahepatic biliary ducts was independent of bowel preparation for two radiologists $\left(P_{1}=\right.$ $\left.0.00276, P_{2}=0.0432, P_{3}=0.51955, P_{4}=0.07524\right)$.

Visualization of extraluminal bowel pathologies was significantly improved in patients with ascites prepared with negative per-oral SPIO contrast agent according to all four radiologists $\left(P_{1}=0.01751, P_{2}=0.03526, P_{3}=0.05304\right.$, $\left.P_{4}=0.00167\right)$ as depicted in Fig. 4. In patients without ascites, the visualization of extraluminal bowel pathologies was independent of bowel preparation.
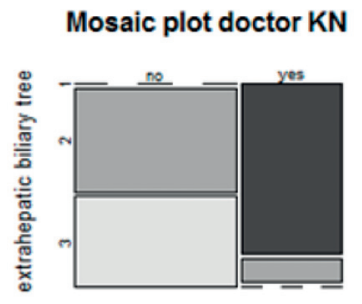

SPIO

Mosaic plot doctor PN

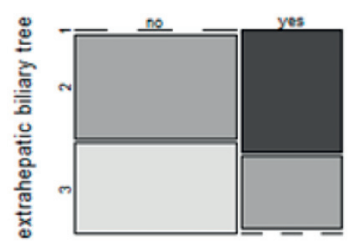

SPIO
Mosaic plot doctor NI

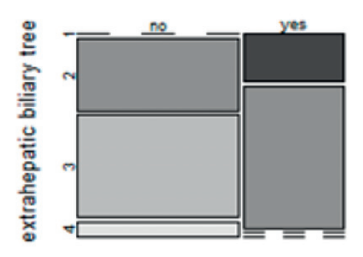

SPIO

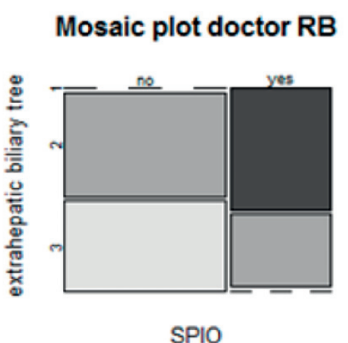

Fig. 1. Mosaic plots-statistic evaluation of extrahepatic biliary duct in patients with ascites in 4 radiologists separately (KN, NI, PN, RB). Mosaic plots as a statistic evaluation of the answers of four radiologists on the given question from the blind questionnaire: "How is the visualization of extrahepatic biliary tree in patients suffering of ascites?" [1. excellent (80-100\%), 2. sufficient (50-80\%), 3. insufficient (less than 50\%), 4. impossible to evaluate].

No means no contrast agent was used; Yes means negative peroral SPIO contrast agent was used before MRCP investigation; numbers 1, 2, 3 and 4 means excellent, sufficient, insufficient and impossible to evaluate and the portions of the grey areas means what is the \% of the answers related to the given number $(1-4)$.

The distribution of the luminal filling was analyzed in group A. The statistical analysis confirmed that 30 min of per-oral drinking of SPIO contrast agent is sufficient time for distribution of suspension through stomach, duodenum and proximal bowel loops $\left(P_{1}=0.01751, P_{2}=\right.$ $\left.0.03526, P_{3}=0.05304, P_{4}=0.00167\right)$, see Fig 5 . In the presence of bowel wall edema the homogeneity of luminal content in patients prepared with negative per-oral SPIO contrast agent was better than without preparation.

There was no significant difference in gender ratio between groups $(P=0.974)$.

\section{DISCUSSION}

The main aim of this study was to find out if there is an improvement of the assessment of extrahepatic and intrahepatic biliary ducts in patients with liver cirrhosis after application of negative per-oral contrast agent.

MRCP is common non-invasive method in patients with advanced liver cirrhosis and advanced ascites. There is however no systematic literature review focused on using MRCP with negative contrast bowel preparation in ascites and advanced liver cirrhosis on MR. More recent applications include 3D sequences and the use of hepa- 


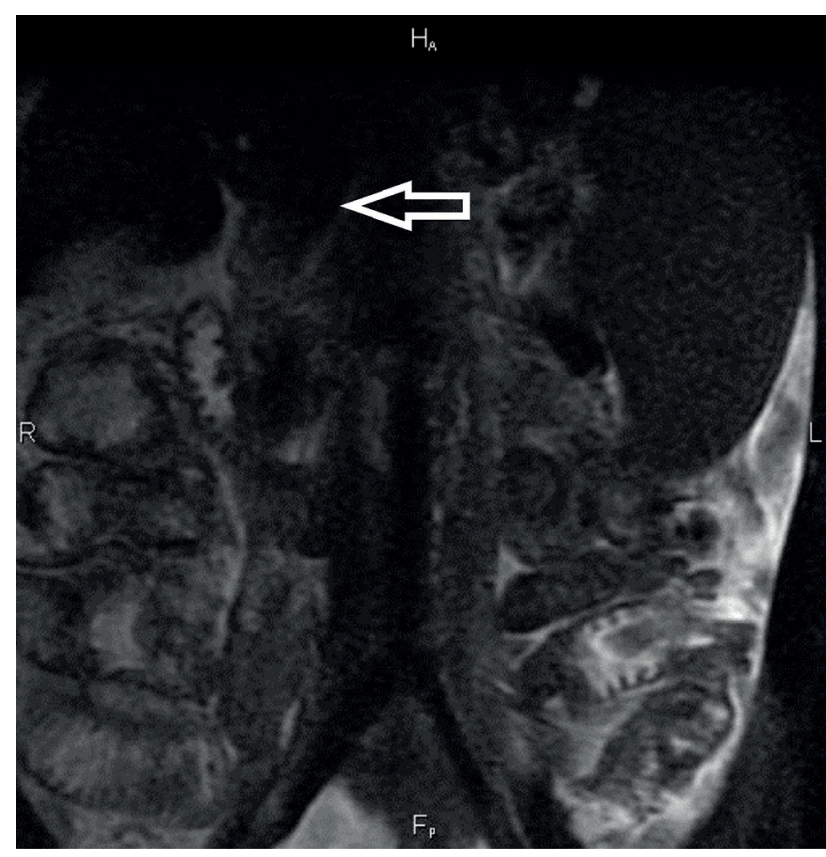

Fig. 2. MRCP + MR enterography without maghemite contrast agent, (after PEG bowel preparation): 52-year-old woman before liver transplantation having liver cirrhosis, due to hepatitis $\mathrm{C}$. Presence of advanced ascites and edema of bowel wall; arrow depicts a delineation of extrahepatic biliary ducts.

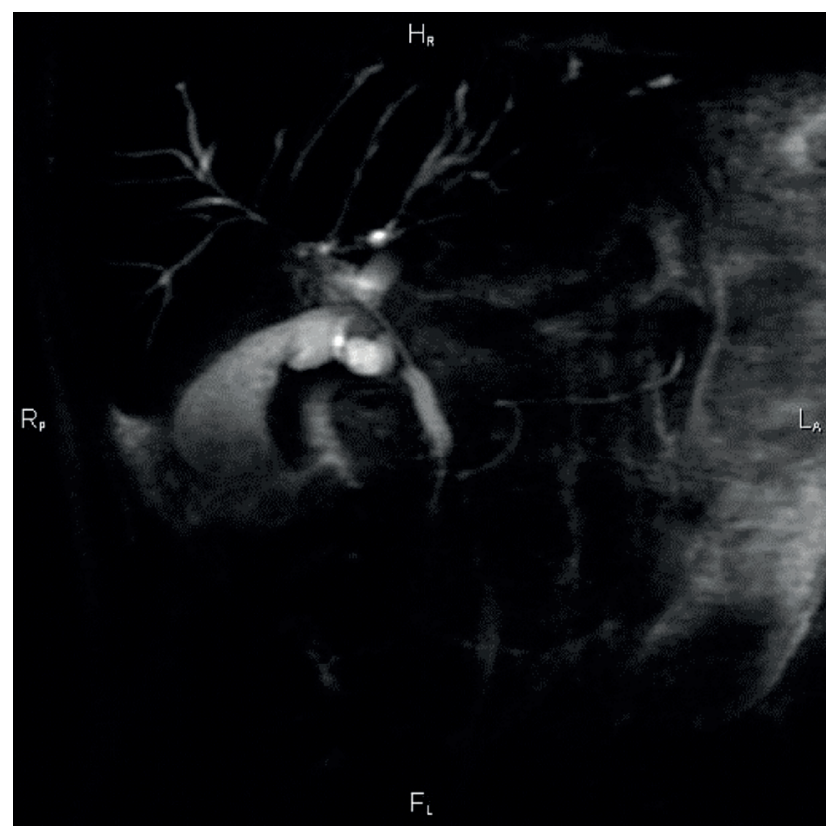

Fig. 4. MRCP with maghemite bowel preparation: 37-year-old man with primary biliary cirrhosis before liver transplantation, presence of advanced ascites. Good delineation of intra- and extrahepatic biliary ducts with multiple stenoses and dilatations and visibility of main pancreatic duct is documented after SPIO per-oral application.

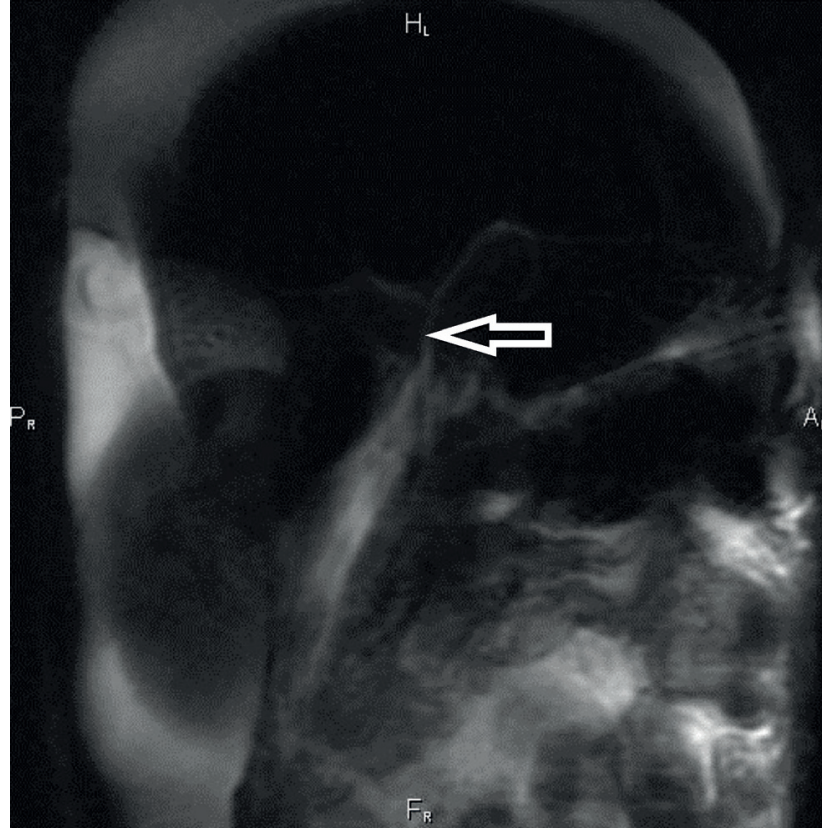

Fig. 3. MRCP + MR enterography with maghemite contrast agent (same patient as in Fig. 2): in cirrhotic liver transformation. Visible improvement of extrahepatic biliary ducts delineation after SPIO per-oral application.

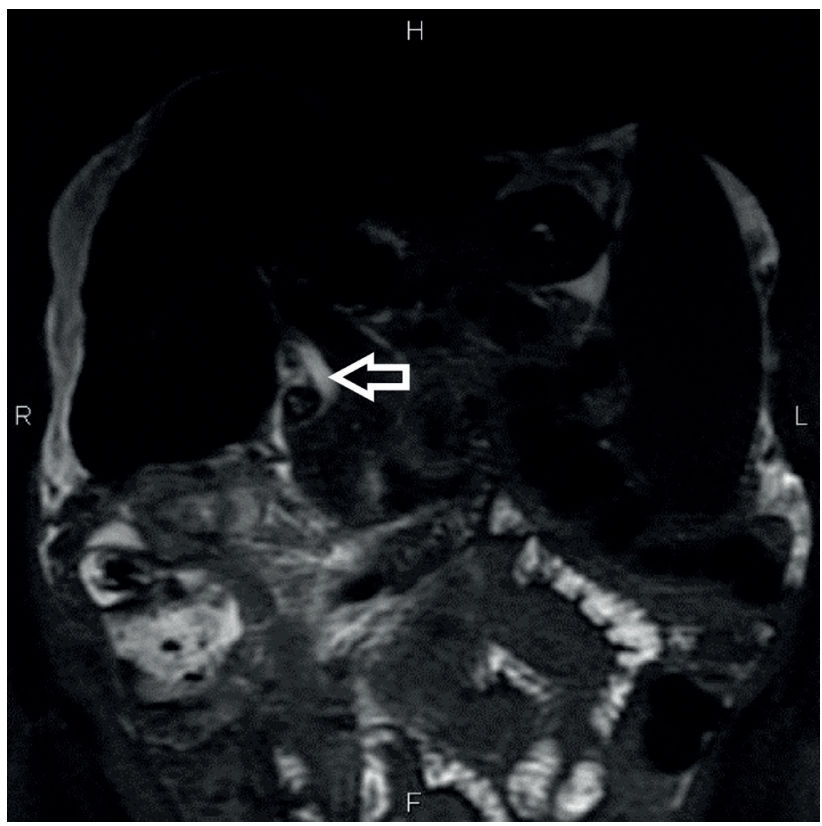

Fig. 5. MRCP with maghemite bowel preparation: 60-year-old woman with advanced liver cirrhosis, before liver transplantation due to primary biliary cirrhosis. Sufficient suppression of the signal of bowel content is seen in the upper part of abdomen leading to better biliary ducts visualization after SPIO per-oral application (arrow). 
to-biliary contrast agents (Primovist) enabling a higher definition of the biliary tree, both under pathological and normal conditions ${ }^{10}$. On the other hand our study was focused on preparation of the upper part of small bowel without using hepato-biliary contrast agent. Hepatobiliary contrast agents are more expensive and are used intravenously in comparison with our per-oral application. Some clinical centers use blueberry or pineapple juices as negative per-oral contrast solutions to reduce the signal intensity of overlapping fluid within the stomach, duodenum, although this is not a part of routine protocol MRCP (ref. ${ }^{11,12}$ ). But, from our own experience, a suppression of signal from bowel is not homogenous in comparison with our per-oral maghemite/bentonite contrast agent.

Another solution is using 3D MRCP with respiratory triggering. This has been shown to be feasible in routine clinical practice and improving free breathing 3D MRCP (ref. ${ }^{13}$ ), as well as in uncooperative patients ${ }^{14}$. Moreover, introduction of $3 \mathrm{~T}$ magnets can provide a better anatomic detail. But no valuable studies recommending these protocols have been published yet.

From our statistical results, we found better extrahepatic biliary duct visualization and visibility of extraluminal pathologies in patients with ascites after SPIO per-oral bowel preparation on 1.5 T MR using 2D MRCP protocol. Fig. 1 depicts the schematic statistical evaluation of the responses of 4 radiologists in the form of Mosaic plots on the given question from the blind questionnaire: "How is the visualization of extrahepatic biliary tree in patients suffering of ascites?" From the graphical plots assessed for each radiologist individually it is observed and demonstrated that for all 4 radiologists the visualization of extrahepatic biliary ducts in patients with ascites was excellent (dark grey) or sufficient (middle gray) after administration of negative per-oral SPIO contrast agent while in the cases without bowel preparation the visualization was found in almost half of patients insufficient (light grey). Visible difference can be seen in Fig. 2 and 3 between patients without and after per-oral SPIO agent administration. In the cirrhotic patient, waiting for liver transplantation, the delineation of extrahepatic biliary ducts was complicated and not accurate in the presence of artefacts and non-homogeneities due to the advanced ascites and edema of bowel wall (Fig. 2). After using SPIO per-oral contrast agent, there was substantial visible improvement of delineation of extrahepatic biliary duct (Fig. 3).

No statistically significant difference was found for visualization of extrahepatic biliary ducts in patients without ascites. We were not able to decide whether visualization of intrahepatic biliary ducts was really independent of bowel preparation or not. In spite of the results, we incline to the opinion that there exists a difference in these two approaches. The main reason is that three of the $\mathrm{p}$-values were significantly under a level of confidence $10 \%$. Fig. 4 depicts the patient with primary biliary cirrhosis before liver transplantation having advanced ascites. MRCP with maghemite bowel preparation was performed and showed good delineation of intra- and extrahepatic biliary ducts with multiple stenoses and dilatations. Extrahepatic biliary ducts and main pancreatic duct were clearly visible.
Our results also confirmed that 30 minutes of per-oral preparation was sufficient for MRCP (Fig. 5).

\section{CONCLUSION}

Our experimentally prepared per-oral SPIO contrast agent based on maghemite/bentonite is useful in MRCP for the improvement of visibility of extrahepatic biliary ducts particularly in patients with ascites. This is a first experimental study which recommends a protocol for diagnosis of extrahepatic biliary ducts in advanced ascites combined with liver cirrhosis on 1.5T MR using per-oral negative contrast agent and thus very helpful for surgeons undertaking liver transplantations.

\section{ABBREVIATIONS}

CT, Computed Tomography; ERCP, Endoscopic Retrograde Cholangiopancreatograohy; FIESTA, Fast Imaging Employing Steady State Acquisition; MELD, Model for End-Stage Liver Disease; MR, Magnetic Resonance; MRCP, Magnetic Resonance Cholangiopancreatography; SI, Signal Intensity; SPIO, Superparamagnetic Iron Oxide; SSFSE, Single Shot Fast Spin Echo; US, Ultrasound.

Acknowledgement: The authors gratefully acknowledge the support by the Operational Program Education for Competitiveness - European Social Fund (project CZ.1.07/2.3.00/20.0170) of the Ministry of Education, Youth and Sports of the Czech Republic.

The authors gratefully acknowledge the support by the project LO1305 and LO1304 of the Ministry of Education, Youth and Sports of the Czech Republic and the Operational Program Education for Competitiveness European Social Fund (project CZ.1.07/2.3.00/20.0155). Author contributions: KP: coordinator, synthesis of contrast agent, literature search, manuscript writing; IM: coordinator, design MRI examinations, data analysis, manuscript writing; DP, PT: statistic data analysis; PN, KN, IN, RB: MRI data analysis; RZ, MH: final approval. Conflict of interest statement: None declared.

\section{REFERENCES}

1. Bruix J, Sherman M, Llovet JM, Beaugrand M, Lencioni R, Burroughs A, Christensen E, Pagliaro L, Colombo M, Rode J. Clinical management of hepatocellular carcinoma. Conclusions of the Barcelona-2000 EASL. J Hepatol 2001;35:421-30.

2. Valls C, Alba E, Cruz M, Figueras J, Andía E, Sanchez A, Lladó L, Serrano T. Biliary complications after liver transplantation: diagnosis with MR cholangiopancreatography. AJR 2005;184:812-20.

3. Maccioni F. Double-contrast magnetic resonance imaging of the small and large bowel: effectiveness in the evaluation of inflammatory bowel disease. Abdom Imaging 2010; 35(1):31-40.

4. Giovagnoni A, Fabbri A, Maccioni F. Oral contrast agents in MRI of the gastrointestinal tract. Abdom Imaging 2002; 27(4):367-75.

5. Kluchova K, Zboril R, Tucek J, Pecova M, Zajoncova L, Safarik I, Mashlan M, Markova I, Jancik D, Sebela M, Bartonkova H, Bellesi V, Novak P, Petridis D. Superparamagnetic maghemite nanoparticles 
from solid-state synthesis. - Their functionalization towards peroral MRI contrast agent and magnetic carrier for trypsin immobilization. Biomaterials 2009; 30:2855-63.

6. Wang Y-XJ, Hussain $\mathrm{SH}$, Krestin GP. Superparamagnetic iron oxide contrast agents: physicochemical characteristics and applications in MR imaging. Eur Radiol 2001;11:2319-31.

7. Narin B, Aja W, Göhde S, Langhorst J, Akgöz H, Gerken G, Rühm SG, Lauenstein TC. Combined small and large bowel MR imaging in patients with Crohn's disease: A feasibility study. Eur Radio 2004;14:1535-42.

8. Maccioni F, Viscido A, Marini M, Caprilli R. MRI evaluation of Crohn's disease of the small and large bowel with the use of negative superparamagnetic oral contrast agents. Abdom Imaging 2002; 27:38493.

9. Botta F, Giannini E, Romagnoli P, Fasoli A, Malfatti F, Chiarbonello B Testa E, Risso D, Colla G, Testa R. MELD scoring system is useful for predicting prognosis in patients with liver cirrhosis and is correlated with residual liver function: a European study. Gut 2003;52:134-93.
10. Maccioni F, Martinelli M, Al Ansari N Kagarmanova A, de Marco V, Zippi M, Marini M. Magnetic resonance cholangiography: past, present and future: A review. Eur Rev Med Pharmacol Sci 2010;14:721-25.

11. Karantanas AH, Papanikolaou N, Kalef-Ezra J, Challa A, Gourtsoyiannis $\mathrm{N}$. Blueberry juice used per os in upper abdominal MR imaging: Composition and initial clinical data. Eur Radiol 2000;10:909-13.

12. Riordan, RD, Khonsari M, Jeffries R, Maskell GF, Cook PG. Pineapple juice as a negative oral contrast agent in magnetic resonance cholangiopancreatography: a preliminary evaluation. Br J Radiol 2004;77(924):991-9.

13. Matsunaga K, Ogasawara G, Tsukano M, Iwadatec $Y$, Inouea Y. Usefulness of the navigator-echo triggering technique for freebreathing three-dimensional magnetic resonance cholangiopancreatography. Magn Reson Imaging 2013;3:396-400.

14. Kim JH, Hong SS, Eun HW, Han JK, Choi BI. Clinical usefulness of free-breathing navigator triggered 3D MRCP in non-cooperative patients: Comparison with conventional breath-hold 2D MRCP. Eur J Radiol 2012;8:513-18. 